\title{
Allogeneic Stem Cell Transplantation with a Novel Reduced Intensity Conditioning Regimen for the Treatment of Patients with Primary Cutaneous T-cell Lymphomas
}

\author{
Maria Stamouli ${ }^{1}$, Konstantinos Gkirkas ${ }^{1}$, Aggeliki Karagiannidi ${ }^{1}$, Theodoros Iliakis ${ }^{2}$, Spiros Chondropoulos ${ }^{1}$, \\ Thomas Thomopoulos ${ }^{1}$, Vassiliki Nikolaou ${ }^{3}$, Vassiliki Pappa ${ }^{1}$, Evangelia Papadavid ${ }^{4}$, Panagiotis Tsirigotis ${ }^{1, *}$,(D) \\ ${ }^{1}$ Hematology Division, 2nd Department of Internal Medicine, Propaedeutic, ATTIKON General University Hospital, National and Kapodistrian \\ University of Athens, Greece \\ ${ }^{2}$ Hematology Division, 1st Department of Internal Medicine, Propaedeutic, LAIKON General Hospital, National and Kapodistrian University of \\ Athens, Greece \\ ${ }^{3} 1$ st Department of Dermatology, Syggros Hospital, National and Kapodistrian University of Athens, Greece \\ ${ }^{4}$ 2nd Department of Dermatology, ATTIKON General University Hospital, National and Kapodistrian University of Athens, Greece
}

\section{ARTICLE INFO}

\section{Article History}

Received 27 March 2021

Accepted 21 May 2021

Keywords

Mycosis fungoides sezary syndrome

cutaneous t-cell lymphomas donor lymphocyte infusion

allogeneic stem cell transplantation graft-versus-host disease graft versus lymphoma

\begin{abstract}
The prognosis of patients with mycosis fungoides (MF) and Sezary Syndrome (SS) varies greatly, from near normal life expectancy in patients with early stage, to a median survival of less than 2 years for those diagnosed with advanced stage disease. Initial response to treatment is almost always followed by relapse and, finally, most of patients enter a phase of advanced multi-drug resistant disease with a short life expectancy after multiple lines of treatment. Allogeneic stem cell transplantation (allo-SCT) is usually limited to patients with advanced disease resistant to multiple treatments. Retrospective registry-based studies have shown increased Non-relapse Mortality (NRM) rates in patients with poor performance status, as well as in patients treated with myeloablative conditioning regimens. Another major limitation of allo-SCT is the increased relapse rate which occurs in nearly $50 \%$ of the cases, and is probably due to the fact that only heavily pretreated patients with advanced disease are referred for allo-SCT. Due to the paucity of data, the ideal conditioning regimen which will provide the maximum therapeutic benefit without the cost of increased NRM is not currently known. In this article we present our experience with a novel regimen in the treatment of patients with advanced MF/SS.
\end{abstract}

(C) 2021 International Academy for Clinical Hematology. Publishing services by Atlantis Press International B.V. This is an open access article distributed under the CC BY-NC 4.0 license (http://creativecommons.org/licenses/by-nc/4.0/).

\section{INTRODUCTION}

Primary cutaneous T-cell lymphomas (CTCL), with the most common histologic subtypes mycosis fungoides (MF) and Sezary Syndrome (SS), are rare neoplastic lymphoid disorders that mainly affect the skin [1].

The prognosis of patients with CTCL depends on disease stage and varies greatly, from near normal life expectancy in patients with early stage, to a median survival of less than 2 years for those diagnosed with advanced stage disease [2]. Several parameters such as age, lactate dehydrogenase (LDH) levels and large cell transformation have a significant impact on the outcome of patients with advanced disease and help in the initial prognostic stratification [3].

Treatment consists of multiple drug and procedures, which range from local treatment with corticosteroids and phototherapy to total skin irradiation and systemic administration of immune modulators such as bexarotene and interferon-a. Initial response is almost always followed by relapse and, ultimately, patients enter a phase of

"Corresponding author. Email: panagtsirigotis@gmail.com

Peer review under responsibility of the International Academy for Clinical Hematology advanced multi-drug resistant disease with a short life expectancy [4]. A proportion of patients with advanced disease and acceptable performance status and no major comorbidities are usually referred for allogeneic stem cell transplantation (allo-SCT) [5].

An important feature of the disease is the poor response to chemotherapy, which is usually characterized by partial remissions of short duration. Allo-SCT offers a curative potential, but at the cost of toxicity such as the occurrence of graft versus host disease and its resultant morbidity and mortality. The therapeutic potential of allo-SCT is mainly attributed to the graft versus lymphoma (GVL) effect, which is mediated by alloreactive donor T-lymphocytes.

An important question is the proper timing of allo-SCT during the course of disease. Clearly allo-SCT is contraindicated in patients with early-stage disease who have a long life expectancy with conventional treatment. On the other hand, allo-SCT is the only treatment modality with a reasonable curative potential for patients in advanced phases of the disease [6]. However, the application of allo-SCT is usually limited to heavily pretreated and to patients with high tumor burden resistant to multiple treatments. Moreover, nonrelapse mortality (NRM) is significantly higher in patients with poor performance status as is usually the case in advanced CTCL [7]. 
Previous studies have shown that the use of myeloablative conditioning is associated with a significant increase in transplant related mortality and should be avoided. However, a major limitation of allo-SCT in the treatment of CTCL still remains the significant relapse rate, which occurs in nearly $50 \%$ of patients, and is probably due to the fact that only heavily pretreated patients with advanced disease are referred for allo-SCT [8].

We present our experience with the use of a novel reduced intensity conditioning in 10 consecutive patients with MF/SS treated in our department during the last 6 years.

\section{PATIENTS AND METHODS}

\subsection{Patients}

Ten patients (five male and five female) with a median age of 59 years (range, 37-71) were included in this study. Two and eight patients had a diagnosis of SS and MF respectively, while two out of 10 patients had large cell transformation in skin lesions at the time of transplant. Patients were heavily pre-treated: the median number of lines of treatment administered before transplant was 4 (range, 3-6). All patients had active disease at the time of transplant which was refractory to the previous treatment in nine out of 10 cases. Patients' characteristics are shown in detail in Table 1.

\subsection{Conditioning Regimen and Graft-versus-Host Disease Prophylaxis}

A reduced intensity conditioning regimen consisting of fludarabine $\left(30 \mathrm{mg} / \mathrm{m}^{2}\right.$, days -7 to -4$)$ and cyclophosphamide $(30 \mathrm{mg} / \mathrm{kg}$, days -5 and -4$)$, was used in all patients. For patients with matched sibling donors (MSD) and well matched (10/10 allele matched) unrelated donors (WMUD), 2 Gy total body irradiation (TBI) was administered on day -1 . For patients with 1-locus HLA mismatch unrelated donors (MMUD) the dose of TBI was increased to $4 \mathrm{~Gy}$. Graft-versus-host disease (GVHD) prophylaxis consisted of cyclosporine (CyA) plus Mycophenolate Mofetil (MMF) for MSD and WMUD. Alemtuzumab $10 \mathrm{mg}$ IV on days -2 and -1 was added to standard GVHD prophylaxis for patients transplanted from MMUD. Written informed consent was obtained from all patients and donors. The donor was MSD in four patients, WMUD in four and MMUD in two. G-CSF mobilized peripheral blood (PBSC) was the source of stem cells in all cases. The median number of CD34+ cells infused with the graft was $7.2 \times 10^{6} / \mathrm{kg}$, (range, 4.8-9.5).

\subsection{Statistical Analysis}

Progression free survival (PFS) was defined as the time from transplant to disease progression or death from any cause. PFS and overall survival (OS) were estimated using the Kaplan-Meier method and log-rank test. Relapse and transplant-associated mortality were competing events and were estimated by using cumulative incidence analysis.

\section{RESULTS}

\subsection{Engraftment and Chimerism}

Neutrophil $(>500 / \mu \mathrm{L})$ and platelet $(>20,000 / \mu \mathrm{L})$ engraftment occurred in all patients at a median of 15 and 12 days after graft infusion, respectively. Secondary graft failure was not observed in any of our patients. Full donor chimerism was achieved in all patients at first evaluation $($ day +60$)$.

\subsection{Acute and Chronic GVHD}

Acute GVHD occurred in four out of 10 patients within a median of 60 days (range, 40-160) after transplant. Organs involved were lower and upper intestinal track in three cases and one case, respectively. Two patients with peak grade II acute GVHD achieved complete remission after initial treatment with corticosteroids, while two patients with severe acute GVHD (grade III and IV) had less than partial response after 5 days of treatment with methylprednisolone $2 \mathrm{mg} / \mathrm{kg}$. Both patients achieved complete remission of GVHD after second line treatment with a combination of ruxolitinib and corticosteroids. In patient no $=10$ acute GVHD occurred as a result of CyA discontinuation at day +50 , because of rapid disease progression manifested as multiple ulcerated skin tumors.

Chronic GVHD occurred in six out of 10 patients within a median of 8 months (range, 5-18) after transplant. Peak grade of chronic GVHD was mild in two patients who never received systemic

Table 1 Patients characteristics at the time of transplant

\begin{tabular}{|c|c|c|c|c|c|c|}
\hline No & Age at TX & Sex & Disease & Stage at TX & Previous treatments & $\begin{array}{l}\text { Disease status at } \\
\text { the time of } \mathrm{TX}\end{array}$ \\
\hline 1 & 66 & $\mathrm{~F}$ & SS & IVA2 & IFN plus Bexarotene, ECP & Untested relapse \\
\hline 2 & 54 & $\mathrm{M}$ & SS & IVA1 & IFN plus Bexarotene, TSEB, Caelyx & Refractory \\
\hline 3 & 59 & $\mathrm{M}$ & MF & IVA2 & PUVA, IFN, Bexarotene, Gemcitabine, Brentuximab, TSEB & Refractory \\
\hline 4 & 55 & $\mathrm{~F}$ & MF & IIB & PUVA, IFN, Bexarotene, Gemcitabine, Brentuximab, TSEB & Refractory \\
\hline 5 & 59 & $\mathrm{~F}$ & MF & IIIA & PUVA, IFN, Gemcitabine, Brentuximab & Refractory LCT $^{*}$ \\
\hline 6 & 37 & M & MF & IIIB & PUVA, IFN, Bexarotene, Gemcitabine, TSEB & Refractory \\
\hline 7 & 67 & $\mathrm{M}$ & MF & IVA1 & PUVA, IFN, Caelyx, CHOP, Chlorambucil, Brentuximab & Refractory \\
\hline 8 & 71 & $\mathrm{~F}$ & MF & IVA1 & PUVA, IFN, Brentuximab, TSEB & Refractory \\
\hline 9 & 59 & $\mathrm{~F}$ & MF & IIB & PUVA, IFN, Brentuximab, Gemcitabine, Caelyx & Refractory LCT ${ }^{*}$ \\
\hline 10 & 57 & $\mathrm{M}$ & MF & IIB & CHOP, Auto-SCT, Caelyx, Gemcitabine & Refractory \\
\hline
\end{tabular}

TX, Transplant; IFN, Interferon; ECP, Extracorporeal photopheresis; TSEB, Total skin electron beam; 'LCT, Large cell transformation in skin lesions. 
immunosuppressive treatment. Moderate chronic GVHD with skin and mouth involvement occurred in two patients, which was completely resolved after treatment with ruxolitinib plus corticosteroids. Severe chronic GVHD occurred in one patient with sclerodermatoid skin changes who is still under treatment with ibrutinib. Another patient developed intestinal vasculitis at 18 months after transplant and died of sepsis as a result of intestinal perforation. Currently only one patient still has active GVHD and receives immunosuppressive treatment. In patients no $=5$ and no $=6$ chronic GVHD developed after therapeutic donor lymphocyte infusion (DLI) administered for treatment of disease progression. GVHD manifestations and outcome are shown in Table 2.

\subsection{Progression Free and Overall Survival}

One patient $($ no $=3$ ) never responded to treatment and died due to progressive disease 3 months after transplantation. Patient no $=4$ developed intestinal vasculitis and died 20 months post-transplant due to intestinal perforation. Another patient $($ no $=6)$ died from heart failure at 51 months after transplant, due to dilated cardiomyopathy diagnosed many years before transplantation.

At 4 months after transplantation complete response (CR) was achieved in five out of 10 patients. One $($ no $=3)$ showed rapidly progressive disease and died. Two patients (no $=5$ and no $=6$ ) with partial remission at 4 months achieved long-lasting CR after therapeutic DLI administration. One patient (no $=9)$ with disease progression at 4 months received therapeutic DLI and achieved CR which is maintained to date (16 months after transplant). Finally, in patient no $=10$, who had disease progression 50 days posttransplant, abrupt CyA discontinuation resulted in CR. Patients' outcome is shown in Table 3.

With a median follow-up of 20 months (range, 2-52) the cumulative incidence of transplant-related mortality (TRM) and disease progression was $12 \%$ and $30 \%$ respectively (Figure 1 ), while the median PFS was 51 months (95\% CI, 2-NA), and the OS was 51 months (95\% CI, 3-NA) (Figure 2).

\section{DISCUSSION}

The prognosis of patients with CTCL is mainly dependent on disease stage. The median OS ranges from 20 years in patients with early stage disease to a median of 2-4 years in those with advanced stage MF and SS. Therapeutic management is mainly based on disease stage. However, the outcome varies greatly within stages and especially among patients with advanced stage disease. In a retrospective study conducted by the Cutaneous Lymphoma International Consortium, data from 1349 patients with advanced stage CTCL were included in a multivariate analysis model. Stage $\mathrm{IV}$, age above 60 years, large-cell transformation in skin lesions and increased LDH were statistically associated with significantly decreased OS. These four variables were used for the development of a prognostic scoring system consisting of three-risk groups with significantly different 5-year OS [3].

There is a relative paucity of data regarding the use of allo-SCT for the treatment of MF/SS. The reluctance of physicians to refer patients for allo-SCT is mainly based on the long-term survival

Table 2 GVHD manifestations and outcome

\begin{tabular}{|c|c|c|c|c|c|c|c|c|c|}
\hline No & Donor & AGVHD & Onset day & Organs & Grade & CGVHD & Onset month & Organs & Grade \\
\hline 1 & WMUD & No & - & - & - & Yes & 12 & Skin & Moderate \\
\hline 2 & MMUD & Yes & 120 & Intestine & II & No & - & - & - \\
\hline 3 & WMUD & No & - & - & - & No & - & - & - \\
\hline 4 & MMUD & Yes & 160 & Intestine & IV & Yes & 18 & Intestine & Severe \\
\hline 5 & MSD & No & - & - & - & Yes $^{*}$ & 5 & Skin & Severe \\
\hline 6 & MSD & No & - & - & - & Yes $^{*}$ & 6 & Skin & Moderate \\
\hline 7 & WMUD & No & - & - & - & Yes & 8 & Skin & Mild \\
\hline 8 & WMUD & Yes & 40 & Stomach & II & Yes & 6 & Oral & Mild \\
\hline 9 & MSD & No & - & - & - & No & - & - & - \\
\hline 10 & MSD & Yes $^{* *}$ & 60 & Intestine & III & N/A & - & - & - \\
\hline
\end{tabular}

”DLI-induced. " Rapid withdrawal of CyA.

Table 3 Patient outcome

\begin{tabular}{|c|c|c|c|c|c|}
\hline No & Response & Relapse & PFS & OS & Comments \\
\hline 1 & CR & No & $+38 \mathrm{~m}$ & $+38 \mathrm{~m}$ & Alive in CR and GVHD free \\
\hline 2 & CR & No & $+34 \mathrm{~m}$ & $+34 \mathrm{~m}$ & Alive in CR and GVHD free \\
\hline 3 & No response & No response & $\mathrm{N} / \mathrm{A}$ & $3 \mathrm{~m}$ & Never responded - died due to infection \\
\hline 4 & CR & No & $20 \mathrm{~m}$ & $20 \mathrm{~m}$ & Died in CR from intestinal vasculitis (GVHD?) \\
\hline 5 & $\mathrm{CR}^{*}$ & No & $+52 \mathrm{~m}$ & $+52 \mathrm{~m}$ & Alive in CR with active sclerodermoid GVHD under treatment \\
\hline 6 & $\mathrm{CR}^{*}$ & No & $51 \mathrm{~m}$ & $51 \mathrm{~m}$ & Died in CR due to dilated cardiomyopathy present before transplantation \\
\hline 7 & CR & No & +24 & +24 & Alive in CR and GVHD free \\
\hline 8 & $\mathrm{CR}$ & No & +20 & +20 & Alive in CR with inactive GVHD under treatment with ruxolitinib \\
\hline 9 & $\mathrm{CR}^{*}$ & Yes & +4 & +16 & Alive in CR and GVHD free \\
\hline 10 & $\mathrm{CR}^{* *}$ & Yes & +2 & +5 & Alive in CR and GVHD free \\
\hline
\end{tabular}

CR achieved after DLI administration. " $\mathrm{CR}$ achieved after CyA withdrawal. 


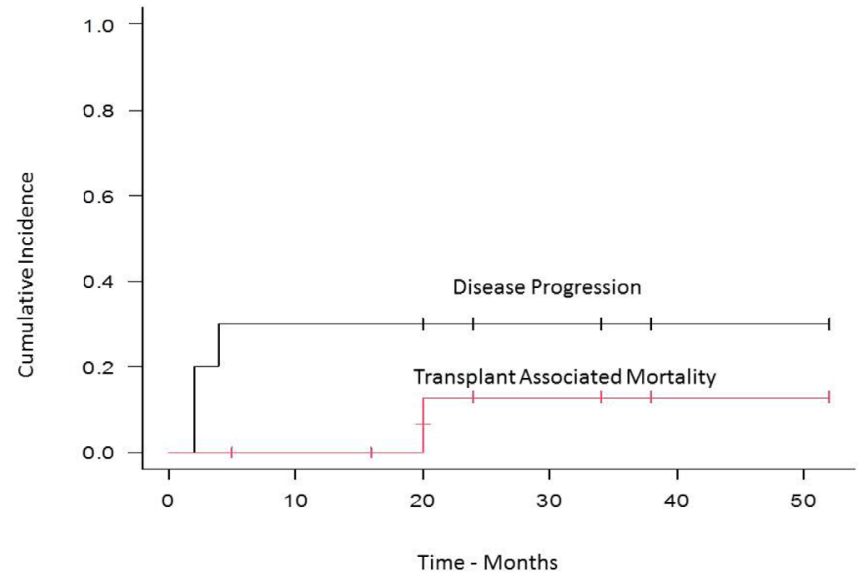

Figure 1 Cumulative incidence of relapse and transplant associated mortality.

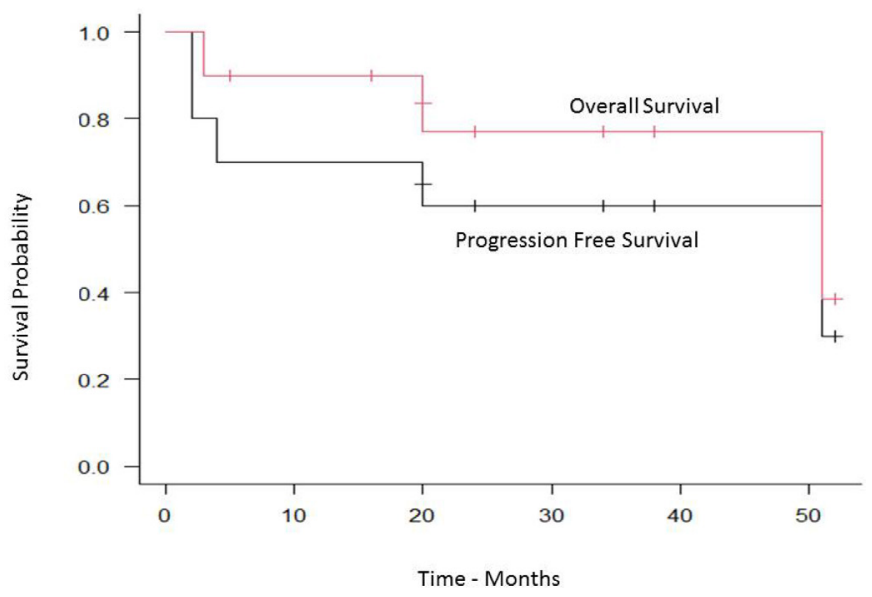

Figure 2 Progression free and overall survival.

observed in early stage disease, and the lack of skin integrity in patients with more advanced disease, raising concerns of high infections rates. Furthermore, the advanced median age and the rarity of the disease explain the small number of patients with CTCL reported in international bone marrow transplantation registries. Published data are mainly based on relatively small retrospective studies of allo-SCT including patients treated with various conditioning regimens and with considerable heterogeneity regarding disease characteristics.

The largest published retrospective study consists of 129 patients reported by the Center for International Blood and Marrow Transplant Research (CIBMTR) between years 2000 and 2009. Myeloablative conditioning was administered in 36\% of patients while a reduced intensity conditioning was used in $64 \%$ of them. Mobilized peripheral blood was the graft source in $83 \%$ of patients. Disease status at the time of transplant was active disease for the vast majority of patients, while $37 \%$ had never achieved CR before allo-SCT. Engraftment occurred in almost all patients, while the 100 -day mortality was $16 \%$. Acute GVHD grade II-IV at 100 days after transplant was experienced by $41 \%$ of patients, while the cumulative incidence of chronic GVHD at 2 years was $43 \%$. Nonrelapse mortality and disease progression occurred in $20 \%$ and $60 \%$ of patients after 3 years, respectively. PFS and OS at 5-years was $32 \%$ and $17 \%$, respectively [9].
In a retrospective study conducted by the Lymphoma Working Party of the European Society for Blood and Marrow Transplantation (EBMT), $60 \mathrm{MF} / \mathrm{SS}$ patients with a median age of 47 years who underwent allo-SCT between 1997 and 2007 and registered in the EBMT database were included in the statistical analysis. The vast majority of patients (73\%) had stage IV disease, with $67 \%$ of them having advanced phase disease defined as more than three previous lines of treatment. A MUD was used in $75 \%$ of cases and a reduced intensity conditioning was administered in $73 \%$ of patients. Graft source was mobilized peripheral blood in $83 \%$ of patients while in approximately half of cases anti-T cell antibodies were administered for GVHD prophylaxis. In multivariate analysis, the use of myeloablative conditioning and poor performance status were independently associated with increased TRM. Advanced phase disease and in vivo T-cell depletion were factors predictive of increased relapse incidence. In the latter study, a significant GVL effect in MF/SS was observed, since a significant number of patients who had disease relapse post-transplant achieved long-lasting responses after DLI administration [10]. These results were recently confirmed in a subsequent analysis after an extended follow-up time [11].

A novel non-myeloablative conditioning has been introduced by researchers in Stanford University for the treatment of patients with CTCL. Conditioning is chemotherapy-free and consists of antithymocyte globulin plus total lymphoid irradiation at a dose of 10-12 Gy administered in 10 fractions. Total skin electron beam irradiation at a dose of 30-36 Gy was initiated 6-9 weeks before the beginning of conditioning in an effort to achieve maximum tumor debulking. Mobilized PBSC were the cell source in all cases, while GVHD prophylaxis consisted of cyclosporine or tacrolimus and MMF [12].

Thirty-five patients with a median age of 62 years were enrolled in the above trial. The vast majority had advanced phase disease at the time of transplant and had been heavily pre-treated. Four and five patients (25\%) had primary and secondary graft failure, respectively. The cumulative incidence of grade II-IV acute GVHD and of moderate/severe chronic GVHD was $16 \%$ and $32 \%$, respectively, while the 2-year NRM was $14 \%$. Twenty out of 35 patients experienced disease relapse/progression post-transplant. With a median follow-up of 5.4 years, the 5-year PFS and OS were $41 \%$ and $56 \%$, respectively. DLI was administered in eight patients for treatment of relapse and, among them, three achieved CR, two achieved partial response, two had stable, while one had progressive disease.

In our study, sustained engraftment was achieved in all patients with the use of a reduced intensity conditioning. The regimen was well tolerated and transplant-related death occurred in only one out of 10 patients. Despite the fact that all patients had active disease at the time of transplant and nine out of 10 were refractory to previous treatment, five out of 10 patients achieved CR at 4 months posttransplant. Persistent remissions are mainly attributed to a powerful GVL effect which is effective in patients with CTCL. As a proof of the concept, therapeutic DLI administration was effective in three out of three patients who achieved long lasting remissions. Moreover, in another patient who progressed after 2 months post-transplant, cyclosporine discontinuation resulted in $\mathrm{CR}$ of the disease.

The limitation of our study is the small number of patients. However, CTCL are rare lymphomas, usually display a long disease course and treating physicians are reluctant to refer them for allo-SCT. As a result, data regarding the efficacy of allo-SCT has been scarce so far and the existing knowledge is mainly based on 
retrospective registry studies. In conclusion, the results of our study are promising and should be tested in larger number of patients.

\section{CONFLICTS OF INTEREST}

The authors declare they have no conflicts of interest.

\section{AUTHORS' CONTRIBUTION}

$\mathrm{PT}$ and MS contributed in conceptualization and design. MS, KG, AK, TI, SC, TT, VN, VP, EP and PT contributed in data collection and writing.

\section{REFERENCES}

[1] Bradford PT, Devesa SS, Anderson WF, Toro JR. Cutaneous lymphoma incidence patterns in the United States: a populationbased study of 3884 cases. Blood 2009;113;5064-73.

[2] Agar NS, Wedgeworth E, Crichton S, Mitchell TJ, Cox M, Ferreira $\mathrm{S}$, et al. Survival outcomes and prognostic factors in mycosis fungoides/Sézary Syndrome: validation of the Revised International Society for Cutaneous Lymphomas/European Organisation for Research and Treatment of Cancer Staging Proposal. J Clin Oncol 2010;28;4730-9.

[3] Scarisbrick JJ, Prince HM, Vermeer MH, Quaglino P, Horwitz S, Porcu P, et al. Cutaneous Lymphoma International Consortium Study of outcome in advanced stages of mycosis fungoides and Sézary Syndrome: effect of specific prognostic markers on survival and development of a prognostic model. J Clin Oncol 2015;33;3766-73.

[4] Trautinger F, Eder J, Assaf C, Bagot M, Cozzio A, Dummer R, et al. European Organisation for Research and Treatment of Cancer consensus recommendations for the treatment of mycosis fungoides/Sézary Syndrome - update 2017. Eur J Cancer 2017;77;57-74.
[5] Larocca C, Kupper T. Mycosis Fungoides and Sézary Syndrome: an update. Hematol Oncol Clin North Am 2019;33;103-20.

[6] Kharfan-Dabaja MA, Kumar A, Ayala E, Hamadani M, Reimer P, Gisselbrecht C, et al. Clinical practice recommendations on indication and timing of hematopoietic cell transplantation in mature T cell and NK/T cell lymphomas: an International Collaborative Effort on Behalf of the Guidelines Committee of the American Society for Blood and Marrow Transplantation. Biol Blood Marrow Transplant 2017;23;1826-38.

[7] Novelli S, Monter A, García-Muret MP, Martino R, Briones J, Sierra J. Discussion on the indication of allogeneic stem cell transplantation for advanced cutaneous $\mathrm{T}$ cell lymphomas. Int J Hematol 2019;110;406-10.

[8] Johnson WT, Mukherji R, Kartan S, Nikbakht N, Porcu P, Alpdogan $\mathrm{O}$, et al. Allogeneic hematopoietic stem cell transplantation in advanced stage mycosis fungoides and Sézary Syndrome: a concise review. Chin Clin Oncol 2019;8;12.

[9] Lechowicz MJ, Lazarus HM, Carreras J, Laport GG, Cutler CS, Wiernik PH, et al. Allogeneic hematopoietic cell transplantation for mycosis fungoides and Sezary Syndrome. Bone Marrow Transplant 2014;49;1360-5.

[10] Duarte RF, Canals C, Onida F, Gabriel IH, Arranz R, Arcese W, et al. Allogeneic hematopoietic cell transplantation for patients with mycosis fungoides and Sézary Syndrome: a retrospective analysis of the Lymphoma Working Party of the European Group for Blood and Marrow Transplantation. J Clin Oncol 2010;28;4492-9.

[11] Duarte RF, Boumendil A, Onida F, Gabriel I, Arranz R, Arcese W, et al. Long-term outcome of allogeneic hematopoietic cell transplantation for patients with mycosis fungoides and Sézary Syndrome: a European society for blood and marrow transplantation lymphoma working party extended analysis. J Clin Oncol 2014;32;3347-8

[12] Weng WK, Arai S, Rezvani A, Johnston L, Lowsky R, Miklos D, et al. Nonmyeloablative allogeneic transplantation achieves clinical and molecular remission in cutaneous T-cell lymphoma. Blood Adv 2020;4;4474-82. 